\title{
A compact all-silicon temperature insensitive filter for WDM and bio-sensing applications
}

\author{
Sarvagya Dwivedi, Herbert D'heer and Wim Bogaerts, Member IEEE
}

\begin{abstract}
We propose a compact, temperature-insensitive, all-silicon Mach-Zehnder interferometer (MZI) filter that uses the polarization-rotating asymmetrical directional couplers. Temperature sensitivity of the filter is less than $8 \mathrm{pm} / \mathrm{K}$ for a wavelength range of $30 \mathrm{~nm}$. The device achieves a reduced footprint by making use of different polarizations, which is made possible by the asymmetric directional couplers that act both as a splitter/combiner and as a polarization rotator. Simulation of the device shows that it can also be useful for gas sensing and bio-sensing applications with 3 times larger response to cladding changes while keeping a thermally robust behavior.
\end{abstract}

Index Terms - Silicon photonics, wavelength filtering devices, athermal, polarization selective devices, bio-sensing

\section{INTRODUCTION}

Silicon has a very high refractive index, enabling optical waveguides with a very high refractive index contrast which opens the possibility of micron-sized devices in silicon photonics. An important limitation for a wide adoption of silicon photonic devices is the high thermo-optic (TO) coefficient of silicon $\left(1.86 \times 10^{-4} \mathrm{~K}^{-1}\right)$. This means that the refractive index changes with the temperature and hence an active tuning is needed to maintain a fixed wavelength operation. Several methods have been proposed to compensate this effect, one of them being the introduction of material(s) with a negative thermo-optic coefficient as cladding, such as polymers [1]. However, it is not always desirable to incorporate such materials in a CMOS-like process flow. Another approach is to use local heaters to dynamically stabilize the device but this approach consumes power and requires supporting logic [2].

For an interferometric device such as a Mach-Zehnder Interferometer (MZI) it is also possible to obtain temperature insensitivity without such measures by combining wide and narrow waveguides in different arms [3,4]. The two arms of the MZI have a different TO coefficient which directly depends upon the confinement in the silicon core. By choosing appropriate arm lengths the temperature dependence of both the arms can be made to cancel each other out. However, this technique increases the device footprint considerably. To reduce the footprint, the difference in the TO coefficient in the two arms should be as large as possible. In [4] this is realized

Manuscript received July 30, 2013

S. Dwivedi, H. D'heer and W. Bogaerts are with the Photonics Research Group, Department of Information Technology, Ghent University-imec, Center of Nano-and Biophotonics, St. Pietersnieuwstraat 41, 9000 Gent, Belgium (E-mail: sarvagya.dwivedi@intec.ugent.be, herbert.dheer@ugent.be and wim.bogaerts@intec.ugent.be). by using a very narrow waveguide in one arm, but the performance of this device is limited by the high propagation loss of the narrow waveguide. In this paper we use the TM polarization in a broader waveguide [5]. Since the TM mode is less confined in the silicon core than the TE mode, this results in a lower TO coefficient. The loss is also lower since the TM mode does not have high field intensities on the rough sidewalls. By exploiting both the polarizations of the waveguides we can achieve similar temperature insensitivity, but at one third of the footprint of [3].

The insensitivity to temperature variation (which is related to a change in the waveguide dimensions) makes the device more sensitive to the changes in the device cladding. Simulations show that the sensitivity to the refractive index change of the cladding of the entire MZI (not just of one arm) is enhanced with respect to a single-polarization device. This makes the device useful as a sensor that is both sensitive to cladding variations (e.g. gas sensing, bio-sensing) and at the same time remains thermally robust.

\section{Design AND Simulations}

In a standard MZI, the Free Spectral Range (FSR) is given by

$$
F S R=\frac{\lambda^{2}}{n_{g} \Delta L}
$$

where $\lambda$ is the center wavelength, $n_{g}$ is the group index and $\Delta L$ is the path length difference between both the arms. For the proposed structure (schematic shown in figure 1) we use different waveguide in the two arms, and the modified expression is

$$
F S R=\frac{\lambda^{2}}{n_{g, T M} \cdot L_{T M}-n_{g, T E} \cdot L_{T E}}
$$

where $n_{g, T M}, n_{g, T E}$ and $L_{T M}, L_{T E}$ correspond to the group index and the length of TM and TE arm respectively.

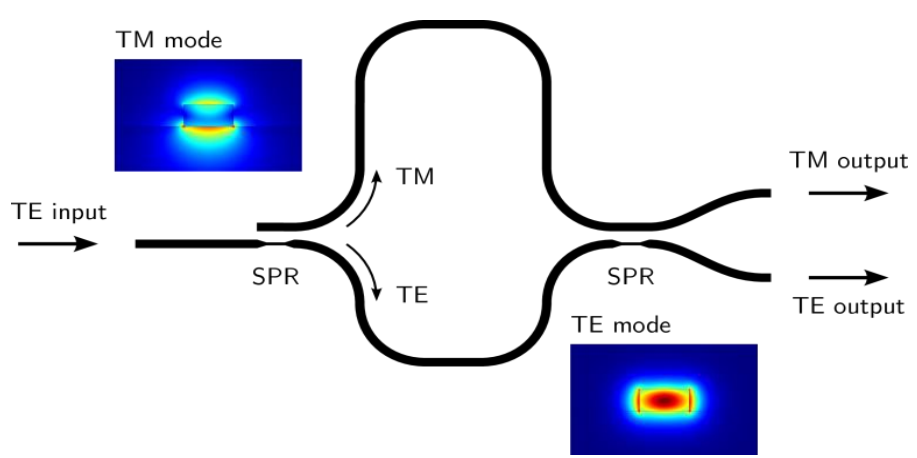

Fig. 1. Schematic of the proposed temperature insensitive filter with TM and TE mode profile 
To make the device athermal the wavelength shift with respect to the temperature should be brought to zero, i.e.

$$
\frac{d \lambda}{d T}=\lambda \frac{T O_{T M} \cdot L_{T M}-T O_{T E} \cdot L_{T E}}{n_{g, T M} \cdot L_{T M}-n_{g, T E} \cdot L_{T E}} \rightarrow 0
$$

Here, $T$ is the temperature and $T O$ is the thermo optic coefficient of the corresponding arms. By combining (2) and (3), the required length of TE and TM arms for any FSR is given by

$$
\begin{aligned}
L_{T E} & =\frac{\lambda^{2}}{\left(n_{g, T M} \frac{T O_{T E}}{T O_{T M}}-n_{g, T E}\right) F S R} \\
L_{T M} & =\frac{\lambda^{2}}{\left(n_{g, T M}-n_{g, T E} \frac{T O_{T M}}{T O_{T E}}\right) F S R}
\end{aligned}
$$

The proposed device shown in figure 1, requires a polarization rotation in one of the arms of the MZI. To accomplish this, we include a customized directional coupler which acts as an integrated splitter polarization-rotator (SPR) which splits the light into two parts and rotates the polarization in one arm. The concept is based on [6] and consists of an asymmetrical directional coupler conditioned for phase a matching between the TE and the TM polarization. The SPR is designed for equal power splitting of the input light in two arms of the MZI filter and then similarly combining the light at the output.

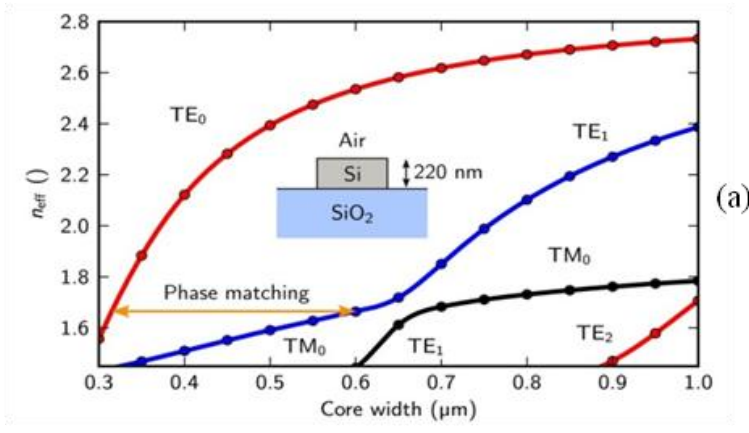

(a)
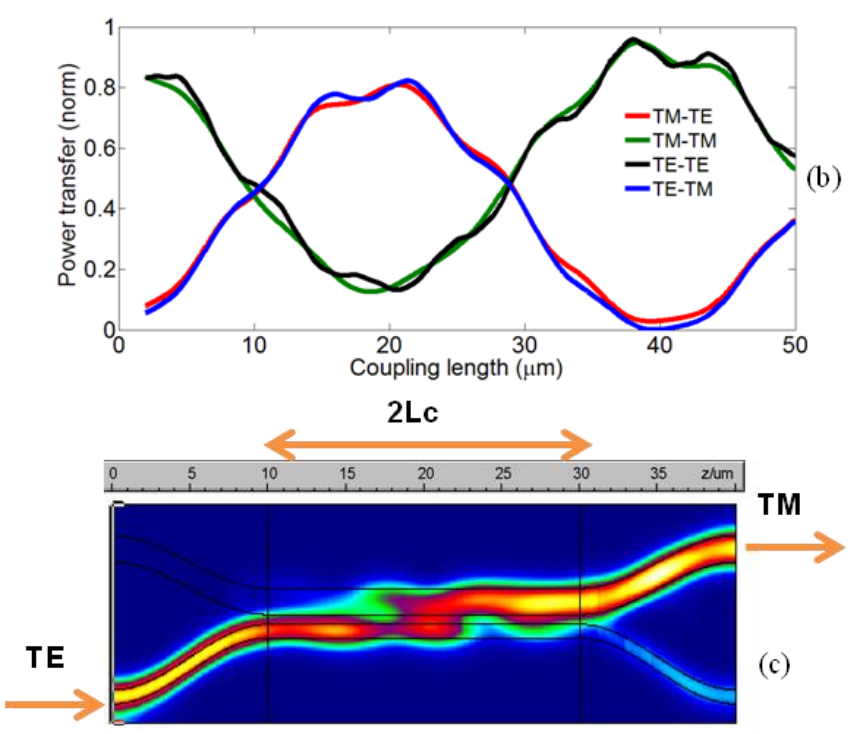

Fig. 2. (a) $n_{\text {eff }}$ variation with waveguide width of a SOI waveguide with air cladding (inset) showing a phase matching condition, Fig. 2(b) Mode beating from one mode to another as a function of coupling length showing the full mode conversion and Fig.2(c) Intensity profile for the calculated cross coupling length from the input TE mode to the output TM mode. All simulations are performed at a wavelength of $1.55 \mu \mathrm{m}$
Figure 2(a) shows the variation of the effective refractive index $\left(n_{e f f}\right)$ with the width of a SOI photonic wire for both the TE and TM mode. The arrow shows the phase matching condition, $\mathrm{n}_{\mathrm{eff}}, \mathrm{TE}=\mathrm{n}_{\mathrm{eff}} \mathrm{TM}$ at a simulated TE width of $320 \mathrm{~nm}$ and TM width of $600 \mathrm{~nm}$. Figure 2(b) shows the simulation of the mode power coupling between the fundamental TE and the fundamental TM mode at the input and output respectively. The optimal coupler length (Lc) for an equal power splitting between the fundamental TE and fundamental TM mode is found to be around $10 \mu \mathrm{m}$. Figure 2(c) shows the full wave simulation of the SPR for the mentioned widths and length Lc. The gap is set as $200 \mathrm{~nm}$. In principle, the SPR length can be reduced by decreasing the gap to $100 \mathrm{~nm}$ but this makes the device less tolerant. The compactness of the MZI athermal filter depends upon the difference in the TO coefficients of the arms. Figure 3 shows the simulated TO coefficient of the TE and TM mode of a SOI waveguide as a function of waveguide width. If the waveguides with the same polarization, but with different widths (i.e. different TO coefficients) are combined, as mentioned in $[3,4]$, the device either suffers from a large footprint penalty (due to the small difference in TO coefficients between the two arms of the device), or from a high insertion loss (high propagation loss in narrow waveguides).

Our solution makes this trade-off easier by using different polarizations in the two arms. TE and TM have a large difference between their TO coefficients and thus a small footprint device is possible. The wider waveguides ensure low-loss and more tolerant behavior to fabrication variations. Fig. 3 shows that a much larger difference than previously reported results [3, 4] in the TO coefficient can be achieved by using two polarizations, even when using waveguides of the same width. For the experimental device, the width of both arms were chosen to be $600 \mathrm{~nm}$ with $\mathrm{n}_{\mathrm{g}, \mathrm{TE}}=4.02$ and $\mathrm{n}_{\mathrm{g}, \mathrm{TM}}=$ 3.73 at $1.55 \mu \mathrm{m}$. Note that an even larger difference is possible by using both polarizations at different widths.

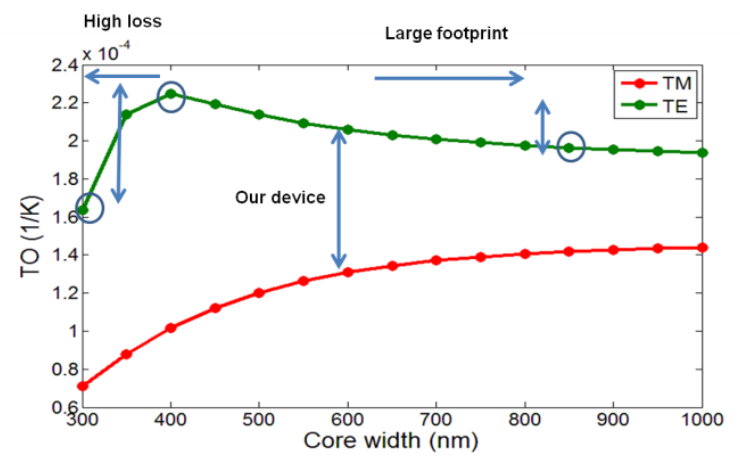

Fig. 3. Simulated thermo-optic coefficients of TE and TM modes of SOI waveguide as a function of core width at a wavelength of $1.55 \mu \mathrm{m}$

\section{FABRICATION}

The device was fabricated in the imec $200 \mathrm{~mm}$ CMOS pilot line, using silicon-on-insulator wafers with $220 \mathrm{~nm}$ of silicon and $2 \mu \mathrm{m}$ of buried oxide. The fabricated device and one of the asymmetrical directional couplers which is fabricated separately are shown in figure 4(a) and 4(b), respectively. The input TE-polarized light passes through the SPR that splits and converts half of the power into TM polarization. The TM light 
goes to the upper arm, while the remaining half of the input power remains in the lower arm as TE polarized light. Finally, both polarizations combine at the MZI output using a similar SPR as a combiner. The device also includes adiabatic tapers before and after the SPR section. The need for an adiabatic taper is to prevent coupling between the fundamental mode and the higher order modes. Compact curved grating couplers [7] designed for the C-band operation, are added to couple light in and out of the device. Depending on the arm, a coupler for either the TE or the TM polarization is used. The length of designed TM arm is $(213.25 \mu \mathrm{m})$ longer than TE arm (139.25 $\mu \mathrm{m}$ ) due to the lower TO coefficient of TM mode (see equation 4.1-4.2). The fabricated length of the SPR is $10 \mu \mathrm{m}$ and the gap is $190 \mathrm{~nm}$. The width of the SPR waveguides is $560 \mathrm{~nm}$ and 310 $\mathrm{nm}$ respectively, as measured from a FIB cross section image. The fabricated width of both the upper TM arm and lower TE arm is $560 \mathrm{~nm}$. Adiabatic tapers are shown in the inset of figure 4(a). Since the upper arm guides the TM mode, larger bends with a radius of $25 \mu \mathrm{m}$ are used in the design for lower radiation loss. We also incorporated separate test structures for the SPR with a sweep of the coupling length and widths.

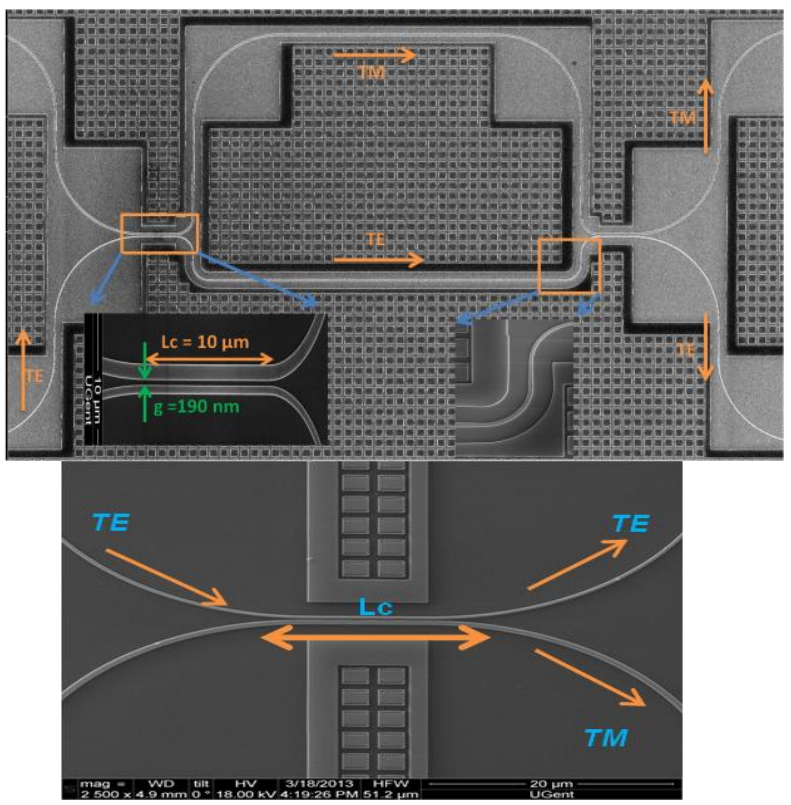

Fig. 4(a) SEM image of fabricated athermal MZI filter with zoomed in SPR and waveguide tapering and 4(b) SPR test structure

\section{MEASUREMENTS}

For the measurements we placed the fabricated chip on a thermally controlled chuck with a resistive heater and temperature sensor. The designed, measured and simulated results of the device transmission and the associated thermal drift during the wavelength sweep are shown in figure 5. The device was designed to be athermal at central wavelength of $1540 \mathrm{~nm}$ as shown in figure 5(a). The simulations of the designed device were performed with our in-house circuit simulator Caphe [8]. Waveguide tapering is also included in the simulation, taking into account the changes in the mode confinement and the TO coefficient. The measured thermal
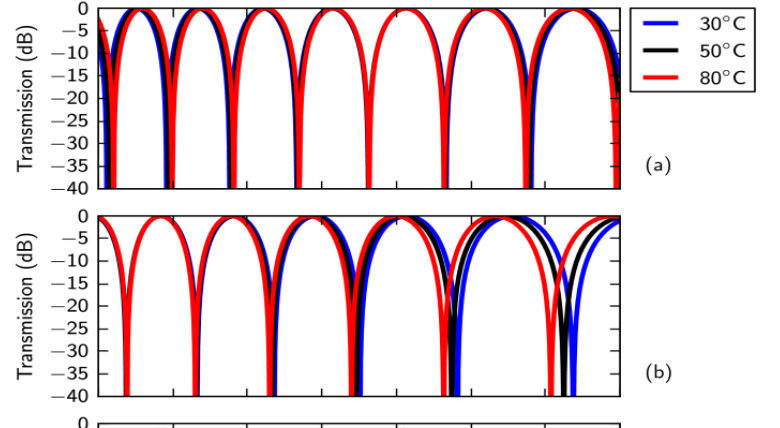

(b)
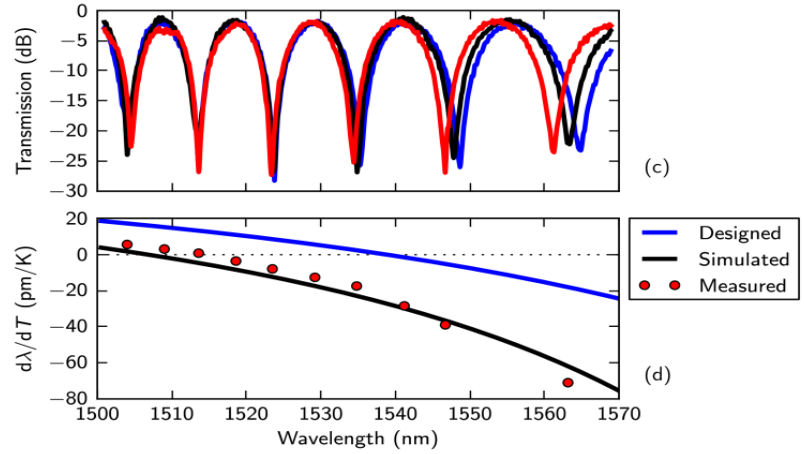

Fig. 5. Designed, simulated and measured transmission spectrum is shown from top to bottom. Thermal sensitivity of these spectrums is shown in the bottom, the athermal region shifted by $20 \mathrm{~nm}$ due to fabrication variation.

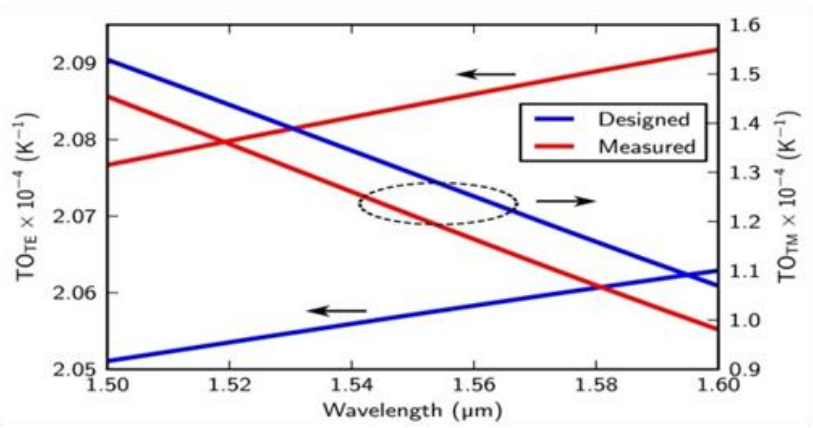

Fig.6. Thermo-optic coefficients of TE and TM mode waveguides for the designed and the measured waveguide widths

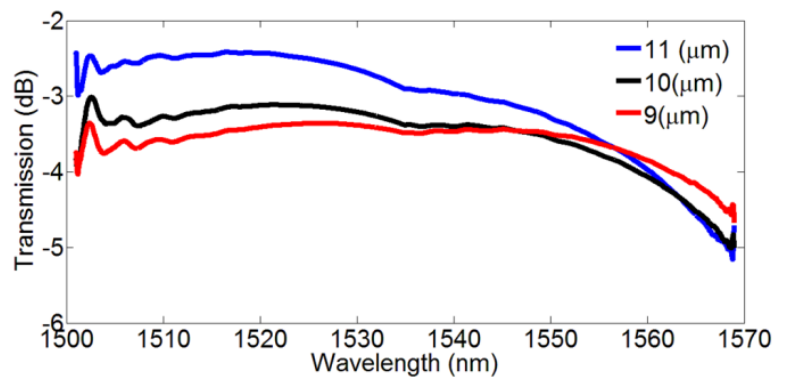

Fig.7. Measured transmission of the SPR test structure for different coupling lengths

sensitivity of the device is less than $8 \mathrm{pm} / \mathrm{K}$ over $30 \mathrm{~nm}$ of wavelength range and is shown in figure 5(c). This matches well with the simulations of the device with the actual (fabricated) geometry, as obtained from the cross-section. These simulations are shown in figure 5(b). We see that, because of the change in the dimensions, the athermal region of the device shifts from $1540 \mathrm{~nm}$ to $1515 \mathrm{~nm}$. Figure 5(d) shows the thermal sensitivity of the device for the original design, the 
fabricated design, and the post-fabricated simulation based on the cross section data. The FSR of the device changes from 9.5 $\mathrm{nm}$ at the lower wavelengths to $13.5 \mathrm{~nm}$ at the longer wavelengths. This is due to the large wavelength dependence of the group index of the TM mode. It is also found that the TO coefficients are strongly wavelength dependent and therefore the device spectrum is red shifted with the increasing temperature for the wavelengths lower than the athermal point, while at longer wavelengths the spectrum is blue shifted. The TO coefficients for the TE and the TM mode of the designed and measured waveguide widths are shown in figure 6.

We also characterized the SPR separately. The transmission spectrum of the SPR for cross coupling is shown in figure 7 for different with varying coupling lengths. It shows that the coupling length of $10 \mu \mathrm{m}$ is closest to the desired $3 \mathrm{~dB}$ of power transfer from the input to the TM mode in the other arm. The variation of $1 \mu \mathrm{m}$ from this length shows a $0.5 \mathrm{~dB}$ variation in transmission in the lower wavelength side of the spectrum. The coupler design is sufficiently tolerant such that a width variation of couple of nanometers in fabrication will not have a detrimental impact. The good splitting ratio also translates into a high extinction ratio (over $25 \mathrm{~dB}$ ) in the measured MZI.

\section{BULK SENSING}

Simulations are performed to test the bio-sensing capabilities of the same device as shown in the figure 8. Bulk refractive index of the cladding is increased from 1.31 to 1.35 of a fluid cladding [9] . DI water TO coefficient is taken from [10]. The simulations were performed for the differential signal: both arms exposed to the same cladding change, as well as the TE arm and TM arm separately, and this was simulated for two different temperatures of $20{ }^{\circ} \mathrm{C}$ and $50{ }^{\circ} \mathrm{C}$ respectively. The absolute athermal wavelength shifted because of the different cladding index (fluid instead of air) but it is still within the C-band.When we increase the cladding index $n_{c}$ on the TE arm only, we get a blue-shifted wavelength response. When we apply the same for the TM arm only, we get a stronger shift, but towards the longer wavelengths. When the cladding of the entire device is changed, then the net response is red-shifted to a lesser degree. The resonance wavelength shifts linearly with cladding the refractive index change. The responsivity of 635 $\mathrm{nm} / \mathrm{RIU}$ is observed in simulation which corresponds to a threefold improvement when compared to a pure TE waveguide. Moreover, the sensor response is temperature independent, and the fact that the entire sensor can be exposed to the analyte can simplify the fluidic design and fabrication. The sensor simulations provided are indicative figures and the device can be improved drastically by designing as a dedicated sensor. The key observation is that the temperature changes do not affect the performance of the sensor.

\section{DISCUSSION AND CONCLUSION}

We demonstrate a compact temperature insensitive MZI filter using a splitter and polarization-rotating section. The measured result shows that the temperature insensitivity is less than $8 \mathrm{pm} / \mathrm{K}$ over a wavelength range of $30 \mathrm{~nm}$. The footprint

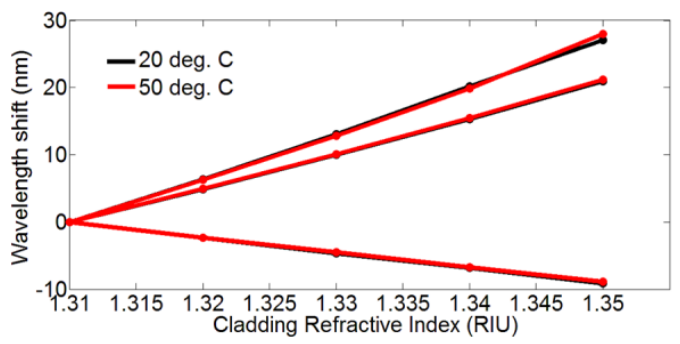

Fig.8. Simulated shift in the resonant wavelength of the separate arms TM, TE and device (differential) with cladding refractive index variation in refractive index unit (RIU) for two different temperatures.

of the device is one-third of the previously reported athermal MZI filter and we show how it can be further reduced. The novel concept gives a lot of flexibility to the designer in terms of choosing waveguide widths with different polarizations without significantly changing the performance of the athermal filter. Simulated results show that the device can also be useful for bio-sensing applications on chip where thermal sensitivity is an issue. These devices can be integrated together to make efficient and compact athermal higher-order filters and de-multiplexers.

\section{ACKNOWLEDGMENT}

The authors acknowledge Martin Fiers for help in circuit simulation software Caphe and Michael Vanslembrouck for measurements support. Part of this work was supported by imec's Optical I/O Core Program.

\section{REFERENCES}

[1] J. Teng, P. Dumon, W. Bogaerts, H. Zhang, X. Jian, X. Han, M. Zhao, G. Morthier, and R. Baets, "Athermal Silicon-on-insulator ring resonators by overlaying a polymer cladding on narrowed waveguides," Opt. Exp., vol. 17, pp. 14627-14633 , Aug. 2009

[2] H. Yu, M. Pantouvaki, S. Dwivedi, P. Verheyen, G. Lepage, R. Baets, W. Bogaerts, P. Absil, J. Van Campenhout, "Compact, Thermally Tunable Silicon Racetrack Modulators Based on an Asymmetric Waveguide," IEEE Phot. Tech. Lett., vol 25, pp. 159-162, Jan. 2013

[3] M. Uenuma, and T. Moooka, "Temperature-independent silicon waveguide optical filter," Opt. Lett., vol. 34, pp. 599-601 , Feb. 2009

[4] B. Guha, A. Gondarenko, and M. Lipson, "Minimizing temperature sensitivity of silicon Mach-Zehnder interferometers," Opt. Exp., vol. 18, pp. 1879-1887, Feb. 2010

[5] S. Dwivedi , H. D'heer and W. Bogaerts, "A compact temperature insensitive filter using polarization-rotating section," in $10^{\text {th }}$ IEEE Int. Conf. Group IV Photonics, 2013.

[6] L. Liu, Y. Ding, K. Yvind, and J. M. Hvam, "Efficient and compact TE-TM polarization converter built on SOI platform with a simple fabrication process ," Opt. Lett., vol.36, pp. 1059- 1061, Apr. 2011

[7] F.V. Laere, T. Claes, J.Schrauwen, S.Scheerlinck, W. Bogaerts, D. Taillaert, L. O'Faolain, D. Van Thourhout and R. Baets, "Compact focusing grating couplers for silicon-on- insulator integrated circuits," IEEE Phot. Tech. Lett., vol 19 , pp. 1919-1921, Dec. 2007

[8] M. Fiers, T. Van Vaerenbergh, K. Caluwaerts, D. Vande Ginste, B. Schrauwen, J. Dambre, P. Bienstman, "Time-domain and frequency-domain modeling of nonlinear optical components on circuit-level using a node-based approach," J. Opt. Soc. Am. B, vol. 29 pp.896-900, May 2012

[9] K. DeVos, I.Bartolozzi, E.Schacht, P. Bienstman, and R. Baets, "Silicon-on-insulator microring resonator for sensitive and label-free biosensing," Opt. Exp., vol. 15, pp. 7610-7615, Jun. 2007

[10] Y.H. Kim, S. J. Park, S. W. Jeon, S. Ju, C. S. Park, W. T. Han an B.H. Lee, "Thermo-optic coefficient measurement of liquids based on simultaneous temperature and refractive index sensing capability of a two-mode fiber interferometric probe," Opt. Exp, vol.20, pp. 23744 - 23754, Oct. 2012 